\title{
Adenosquamous cell lung cancer successfully treated with gefitinib: A case report
}

\author{
KOICHI KURISHIMA ${ }^{1}$, GEN OHARA ${ }^{1}$, KATSUNORI KAGOHASHI ${ }^{1}$, HIROKO WATANABE ${ }^{1}$, \\ NORIO TAKAYASHIKI ${ }^{2}$, ATSUSHI ISHIBASHI ${ }^{3}$ and HIROAKI SATOH ${ }^{1}$ \\ Divisions of ${ }^{1}$ Respiratory Medicine, ${ }^{2}$ Pathology and ${ }^{3}$ Surgery, \\ Mito Medical Center, University of Tsukuba, Mito, Ibaraki 310-0015, Japan
}

Received October 12, 2013; Accepted December 2, 2013

DOI: $10.3892 / \operatorname{mco} .2013 .221$

\begin{abstract}
Although adenosquamous cell lung cancer (ASCLC) is included in the non-small-cell lung cancers (NSCLCs), the number of currently available studies on the response of this type of cancer to epidermal growth factor receptor-tyrosine kinase inhibitors (EGFR-TKIs) is limited. This is the case report of a 66-year-old female who was referred to the Mito Medical Center (Mito, Japan) with hemoptysis and the chest computed tomography (CT) scan revealed a large cavitary mass in the lower lobe of the left lung. The patient underwent surgical resection of the lesion and the final pathological diagnosis was ASCLC staged as pT2bN2M0. Notably, an EGFR exon 19 deletion was identified in the adenocarcinomatous as well as the squamous cell carcinomatous components of the tumor. Despite adjuvant chemotherapy, the patient developed small cavitary metastases in the lungs bilaterally. Therefore, treatment with gefitinib was initiated. The chest CT scan revealed substantial regression of the metastatic cavitary tumors in both lungs, with thinning of the walls. The patient remains alive and recurrence-free 19 months following the initiation of gefitinib therapy. This case demonstrated an optimal clinical response to gefitinib treatment for EGFR mutation-positive ASCLC, suggesting that gefitinib is a therapeutic option for such a subset of patients with ASCLC.
\end{abstract}

\section{Introduction}

Gefitinib, which is one of the highly promising epidermal growth factor receptor-tyrosine kinase inhibitors (EGFR-TKIs), is administered orally once daily for patients with non-small-cell lung cancer (NSCLC) (1). Among NSCLCs, adenosquamous cell lung cancer (ASCLC) is a morphologically mixed type

Correspondence to: Professor Hiroaki Satoh, Division of Respiratory Medicine, Mito Medical Center, University of Tsukuba, Miya-machi 3-2-7, Mito, Ibaraki 310-0015, Japan

E-mail: hirosato@md.tsukuba.ac.jp

Key words: adenosquamous cell lung cancer, gefitinib, cavitary formation, non-small cell lung cancer of tumor, including two cell components, adenocarcinoma and squamous cell carcinoma, in varying proportions, each representing $\geq 10 \%$ of the entire tumor (2). Previous studies evaluated the possibility of monoclonality and similar biological characteristics, including the frequency of EGFR mutation, of the two components (3-12). However, the number of currently available studies on the response of EGFR-positive ASCLC to gefitinib therapy is limited (13). We herein report a case of metastatic ASCLC successfully treated with gefitinib.

\section{Case report}

A 66-year-old female, with no smoking history, presented at the Mito Medical Center (Mito, Japan) with a cough and intermittent hemoptysis over the past 6 weeks. Chest radiography and computed tomography (CT) revealed a large cavitary mass in the lower lobe of the left lung (Fig. 1). Bronchoscopy revealed a tumor occluding the left lower bronchus, with active bleeding. The patient was initially diagnosed with adenocarcinoma on the basis of histopathological examination of transbrochial biopsy specimens. Distant metastasis was not detected. The patient's Eastern Cooperative Oncology Group performance status score was 1 . In order to prevent deterioration of the respiratory condition due to hemoptysis, the patient underwent lobectomy of the left lower lung and mediastinal lymph node dissection. The resected tumor exhibited adenocarcinomatous and squamous cell carcinomatous components, with each comprising $\geq 10 \%$ of the tumor (Fig. 2). The final pathological diagnosis was ASCLC staged as pT2bN2M0. The two components of the surgically resected tumor were precisely separated by manual microdissection under microscopic observation, to avoid contamination of each sample from different components. An EGFR exon 19 deletion was identified in both components. Soon after the surgical resection, the patient received four courses of chemotherapy with carboplatin and pemetrexed. However, six months after the chemotherapy, small cavitary metastases $(\leq 10 \mathrm{~mm})$ were identified in the lungs bilaterally (Fig. 3). Therefore, treatment with $250 \mathrm{mg}$ of gefitinib once daily was initiated. A chest CT scan on day 28 of gefitinib administration revealed a substantial regression of the metastatic cavitary tumors in both lungs, with thinning of the walls (Fig. 4). There were no severe side-effects, such as lung toxicity. Treatment with gefitinib was 


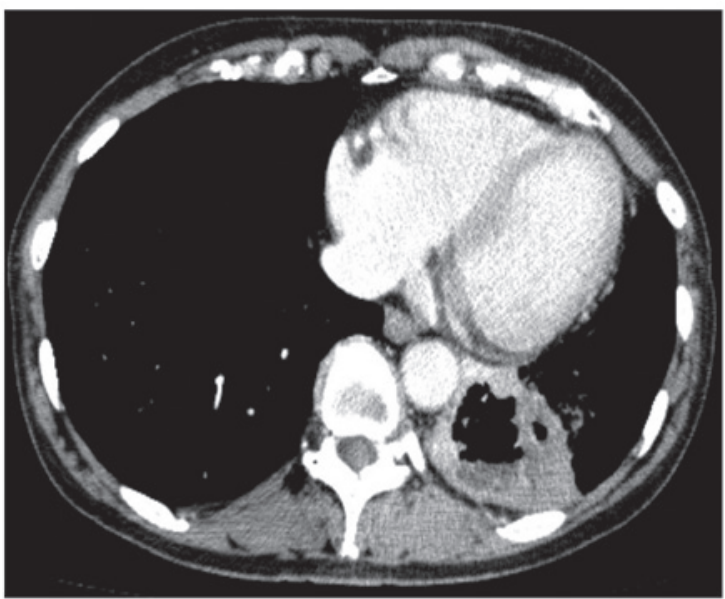

Figure 1. Chest computed tomography scan revealed a large cavitary mass in the lower lobe of the left lung.
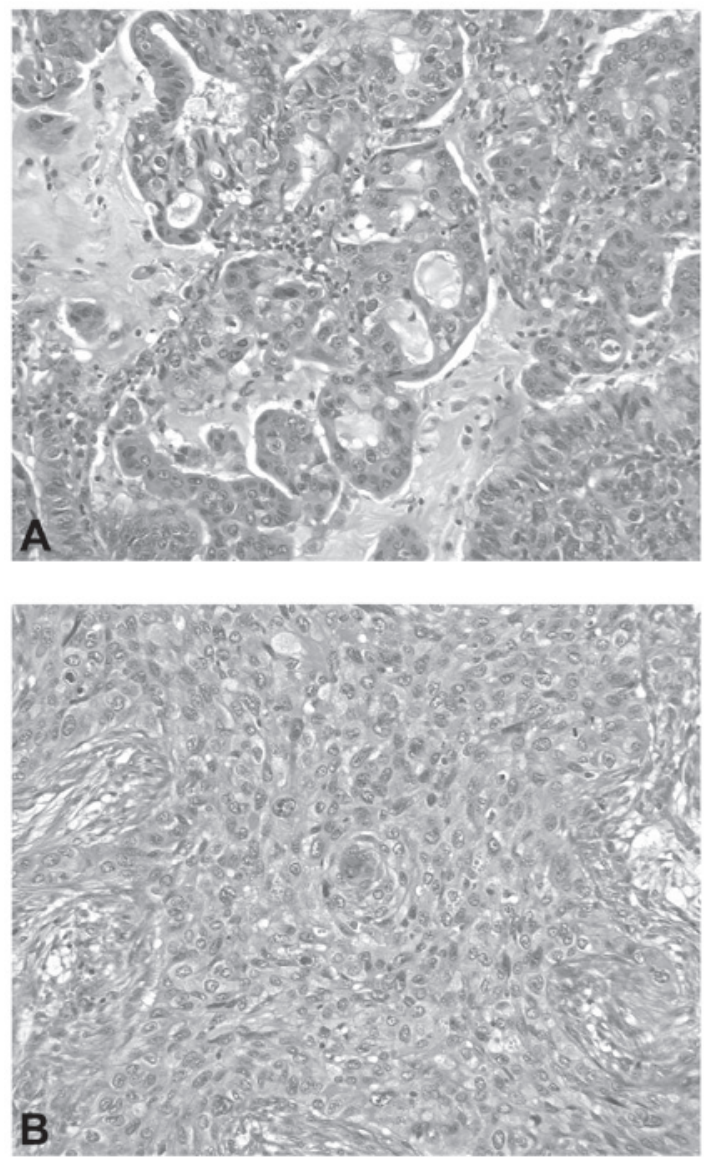

Figure 2. The resected tumor exhibited components of (A) adenocarcinoma and (B) squamous cell carcinoma, with each comprising $\geq 10 \%$ of the tumor.

continued in the outpatient clinic without any signs of tumor progression 19 months after the initiation of the treatment.

\section{Discussion}

Lung cancer is the leading cause of cancer-related mortality worldwide (14). NSCLCs are the most frequent type of lung tumors, with two major histological subtypes: adenocarcinomas

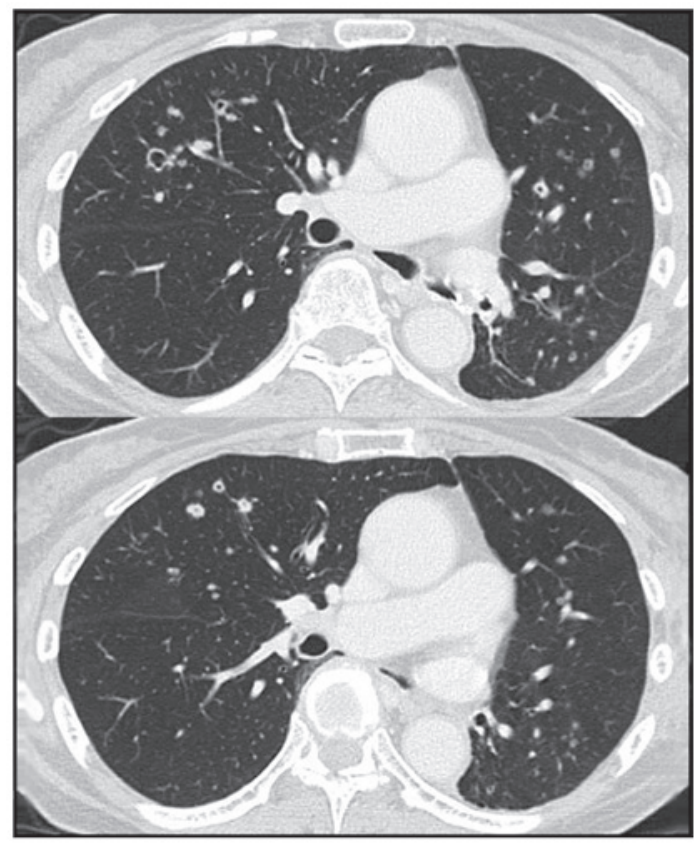

Figure 3. Chest computed tomography scan 6 months after the initiation of chemotherapy revealed small cavitary metastases $(\leq 10 \mathrm{~mm})$ in the lungs bilaterally.

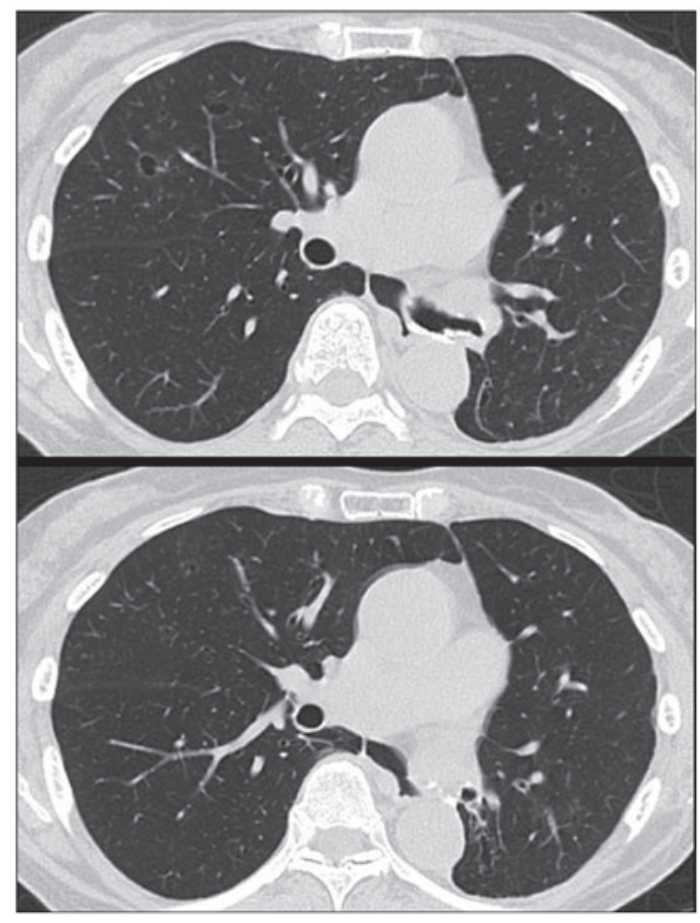

Figure 4. Chest computed tomography scan on day 28 of gefitinib administration revealed substantial regression of the metastatic cavitary tumors in the lungs bilaterally, with thinning of the walls.

and squamous cell carcinomas. A less frequent subtype of NSCLCs, ASCLC, is a morphologically mixed type of tumor, including two cell components, adenocarcinoma and squamous cell carcinoma, in varying proportions, each representing $\geq 10 \%$ of the entire tumor (2). Previous studies have suggested that ASCLC represents $0.3-5 \%$ of NSCLCs $(15,16)$. In certain ASCLC patients, cavitary formation was observed $(17,18)$. 
Kazerooni et al (18) reported that four of 30 cases exhibited cavitary formation. Cavities may be formed as a result of a unidirectional check-valve mechanism (17). Another mechanism of cavity formation may depend on the ischemic or colliquative tumor necrosis associated with neutrophil infiltration into the central portion of lesion. Cavitary formation due to tumor necrosis is common in squamous cell carcinomas, particularly in those developing peripherally in the lung (19). In our patient, the primary lesion exhibited cavitary formation and its wall was composed of the squamous cell carcinomatous component, which was pathologically confirmed. Certain metastatic lesions $\leq 10 \mathrm{~mm}$ also exhibited cavitation. Notably, the wall of the cavities, which were composed of the squamous cell carcinomatous component, were thinned with gefitinib therapy, although the cavities themselves did not change in size in the majority of the pulmonary lesions. The frequency of EGFR mutation-positive ASCLC patients was previously reported (3-12). However, the number of studies on the response of the lesions, either shrinkage or disappearance of pulmonary metastases with cavitary formation, specifically to gefitinib therapy is limited (13). Furthermore, there has been no report on long-term survivors who were successfully treated with gefitinib. Recently, Shukuya et al (13) reviewed the efficacy of gefitinib for non-adenocarcinoma NSCLC patients, including two ASCLC patients, and reported a response rate of $50 \%$, a disease control rate $100 \%$ and a median progression-free survival of 5.3 months (13).

Although ASCLC is included in NSCLCs, the frequency of EGFR abnormalities has not been completely evaluated, due to the rarity of ASCLC (3-12). Two previous studies (4,5), reported EGFR abnormalities in ASCLC tumors identical to those previously described in primary lung adenocarcinoma: Ohtsuka et al (4) identified EGFR TK-domain gene mutations in $2(50 \%)$ of 4 patients with ASCLC, whereas Kang et al (5) identified EGFR mutations in 11 (44\%) of 25 ASCLC patients. However, Sasaki et al (11) reported that only four (15.4\%) of 26 patients with ASCLC were positive for EGFR mutation.

The possibility of monoclonality and similar biological characteristics regarding genetic alterations, chromosomal abnormalities and immunohistochemical reactions in the two components of ASCLCs were evaluated in previous studies (3-12). With regard to EGFR mutations, Kang et al (5) demonstrated identical changes in the two components of ASCLC tumors, with of the nine mutations in 11 ASCLC patients being located in exon 19 (5). Furthermore, Toyooka et al (8) reported that three (27\%) of the 11 ASCLC patients harboured EGFR mutations (two mutations in exon 19 and one in exon 21), which were identical in the two components. In our case, an EGFR mutation (exon 19 deletion) was identified in the two components of the surgically resected tumor. Taken together, the results of previous studies and our findings indicated that TKIs may be a reasonable therapeutic option for ASCLC patients harbouring EGFR mutations. Furthermore, identical EGFR mutations in the adenocarcinomatous and squamous cell carcinomatous components suggest the possibility of monoclonality and similar biological characteristics of the two components.

The present study suggested that gefitinib is a viable therapeutic option for EGFR mutation-positive patients with
ASCLC. However, further investigations into the molecular determinants of tumor monoclonality in the histogenesis of ASCLC and response to EGFR-targeted therapies in patients with ASCLC are required.

\section{References}

1. D'Incecco A and Cappuzzo F: Gefitinib for non-small-cell lung cancer treatment. Expert Opin Drug Saf 10: 987-996, 2011.

2. Travis WD, Brambilla E, Muller-Hermelink HK and Harris CC (eds): Pathology and Genetics: Tumours of the Lung, Pleura, Thymus and Heart. IARC Press, Lyon, 2004.

3. Qin BM, Chen X, Zhu JD and Pei DQ: Identification of EGFR kinase domain mutations among lung cancer patients in China: implication for targeted cancer therapy. Cell Res 15: 212-217, 2005.

4. Ohtsuka K, Ohnishi H, Fujiwara M, et al: Abnormalities of epidermal growth factor receptor in lung squamous-cell carcinomas, adenosquamous carcinomas, and large-cell carcinomas: tyrosine kinase domain mutations are not rare in tumors with an adenocarcinoma component. Cancer 109: 741-750, 2007.

5. Kang SM, Kang HJ, Shin JH, et al: Identical epidermal growth factor receptor mutations in adenocarcinomatous and squamous cell carcinomatous components of adenosquamous carcinoma of the lung. Cancer 109: 581-587, 2007.

6. Niho S, Yokose T, Kodama T, Nishiwaki Y and Mukai K: Clonal analysis of adenosquamous carcinoma of the lung. Jpn J Cancer Res 90: 1244-1247, 1999.

7. Kanazawa H, Ebina M, Ino-Oka N, et al: Transition from squamous cell carcinoma to adenocarcinoma in adenosquamous carcinoma of the lung. Am J Pathol 156: 1289-1298, 2000.

8. Toyooka S, Yatabe Y, Tokumo M, et al: Mutations of epidermal growth factor receptor and K-ras genes in adenosquamous carcinoma of the lung. Int J Cancer 118: 1588-1590, 2006.

9. Huang SF, Liu HP, Li LH, et al: High frequency of epidermal growth factor receptor mutations with complex patterns in non-small cell lung cancers related to gefitinib responsiveness in Taiwan. Clin Cancer Res 10: 8195-8203, 2004.

10. Kosaka T, Yatabe Y, Endoh H, Kuwano H, Takahashi T and Mitsudomi T: Mutations of the epidermal growth factor receptor gene in lung cancer: biological and clinical implications. Cancer Res 64: 8919-8923, 2004

11. Sasaki H, Endo K, Yukiue H, Kobayashi Y, Yano M and Fujii Y: Mutation of epidermal growth factor receptor gene in adenosquamous carcinoma of the lung. Lung Cancer 55: 129-130, 2007.

12. Mitsudomi T, Kosaka T, Endoh $\mathrm{H}$, et al: Mutations of the epidermal growth factor receptor gene predict prolonged survival after gefitinib treatment in patients with non-small-cell lung cancer with postoperative recurrence. J Clin Oncol 23: 2513-2520, 2005.

13. Shukuya T, Takahashi T, Kaira R, et al: Efficacy of gefitinib for non-adenocarcinoma non-small-cell lung cancer patients harboring epidermal growth factor receptor mutations: a pooled analysis of published reports. Cancer Sci 102: 1032-1037, 2011.

14. Alberg AJ, Brock MV and Samet JM: Epidemiology of lung cancer: looking to the future. J Clin Oncol 23: 3175-3185, 2005.

15. Ruffini E, Rena O, Oliaro A, et al: Lung tumors with mixed histologic pattern. Clinico-pathologic characteristics and prognostic significance. Eur J Cardiothorac Surg 22: 701-707, 2002.

16. de Jong WK, Schaapveld M, Blaauwgeers JL and Groen HJ: Pulmonary tumours in the Netherlands: focus on temporal trends in histology and stage and on rare tumours. Thorax 63: 1096-1102, 2008.

17. Sugimoto Y, Semba H, Fujii S, Furukawa E and Kurano R: Clinical analysis of primary lung cancer with a thin-walled cavity to explain the mechanism of thin-walled cavity formation. Nihon Kokyuki Gakkai Zasshi 45: 460-464, 2007 (In Japanese).

18. Kazerooni EA, Bhalla M, Shepard JA and McLoud TC: Adenosquamous carcinoma of the lung: radiologic appearance. AJR Am J Roentgenol 163: 301-306, 1994.

19. Honda O, Tsubamoto M, Inoue A, et al: Pulmonary cavitary nodules on computed tomography: differentiation of malignancy and benignancy. J Comput Assist Tomogr 31: 943-949, 2007. 